\title{
Consensus Analysis for High-Order Multi-Agent Systems without or with Delays
}

\author{
Zhengxin Wang ${ }^{1}$ and Yang $\mathrm{Cao}^{2}$ \\ ${ }^{1}$ College of Science, Nanjing University of Posts and Telecommunications, Nanjing 210003, China \\ ${ }^{2}$ College of Telecommunications and Information Engineering, Nanjing University of Posts and Telecommunications, \\ Nanjing 210003, China
}

Correspondence should be addressed to Zhengxin Wang; zhengxinwang2008@gmail.com

Received 26 August 2013; Accepted 7 September 2013

Academic Editor: Guanghui Wen

Copyright (C) 2013 Z. Wang and Y. Cao. This is an open access article distributed under the Creative Commons Attribution License, which permits unrestricted use, distribution, and reproduction in any medium, provided the original work is properly cited.

\begin{abstract}
This paper studies the consensus problem for a high-order multi-agent systems without or with delays. Consensus protocols, which only depend on the own partial information of agents and partial relative information with its neighbors, are proposed for consensus and quasi-consensus, respectively. Firstly, some lemmas are presented, and then a necessary and sufficient condition for guaranteeing the consensus is established under the consensus protocol without delays. Furthermore, communication delays are considered. Some necessary and sufficient conditions for solving quasi-consensus problem with delays are obtained. Finally, some simulations are given to verify the theoretical results.
\end{abstract}

\section{Introduction}

In recent years, the consensus problem of multi-agent systems has received a great deal of attention due to its broad applications in formation control of unmanned air vehicles, the design of sensor networks, the cooperative control of mobile robots, and automated highway system. In a word, we say all agents reach the consensus upon certain quantities of interest if all agents agree on a common state eventually; the precise definition will be introduced in the following sections. Many researches have been devoted to the consensus problem of multi-agent systems. In 1995, Vicsek et al. [1] proposed a discrete-time model and concluded that all the headings of the agents converged to a common value by simulations. In 2003, Jadbabaie et al. [2] provided a theoretical explanation for Vicsek's simulation results by graph theory. Gradually, the consensus problem of multi-agent systems received a growing attention. For example, Olfati-Saber and Murray [3] discussed consensus problems for networks of dynamic agents with fixed and switching topologies under three cases. Ren and Beard [4] considered the problem of information consensus under dynamically changing interaction topologies and weighting factors. Recently, the second-order consensus problem of multi-agent systems has attracted more and more researchers' attention [5-14]. Different from [5-7, 10], Yu et al. [9], Song et al. [11], and Wen et al. [14] all studied the second-order consensus problem of multi-agent systems with nonlinear dynamics. Compared with synchronization in complex networks which are of nonlinear dynamics, a generalized consensus was investigated. From a unified viewpoint, [15] provided a novel framework for the consensus of multi-agent systems and the synchronization of complex networks. Viewed from the leader-following consensus, both Song et al. [11] and Zhu and Cheng [12] considered the consensus problem with a leader. In general, the second-order consensus problem pays attention to whether the relative position and velocity of each agent will converge to common state.

At the same time, the high-order consensus problem of multi-agent systems is also worth being studied. Generally speaking, the first-order consensus problem of multi-agent systems can be considered as a special case of the synchronization of complex dynamic networks. Moreover, secondorder consensus problem of multi-agent system means that 
the relative position and velocity of each agent will be converged to common. However, the birds achieving the consensus should be on the acceleration besides the relative position and velocity for the flocks of birds [16], which means that all the position, velocity, and acceleration will be converged to common. Recently, there are more and more studies on the high-order consensus or swarming problem (see [1620 ] and the references therein).

Motivated by the previously mentioned results, consensus of high-order multi-agent systems is investigated in this paper. A new consensus protocol which only depends on the own partial information of agents and partial relative information with its neighbors is proposed. In other words, the consensus protocol in this paper does not need the global information of agents and the global relative information. It is convenient to be designed when the global information states cannot be available. Furthermore, we consider the quasi-consensus problem of high-order multi-agent systems with a time-delay protocol. The delay is nonnegligible in the information exchanges due to the limited transmission speed or memory effect. Therefore, there are many papers focusing on the consensus problem with delay [21-25]. It is worth noting that $\mathrm{Yu}$ et al. [25] considered the quasi-consensus problem of second-order multi-agent systems with delay under the protocol

$$
\begin{aligned}
u_{i}(t)= & \alpha \sum_{j=1, j \neq i}^{N} G_{i j}\left(x_{j}(t)-x_{i}(t)\right) \\
& -\alpha \sum_{j=1, j \neq i}^{N} G_{i j}\left(x_{j}(t-\tau)-x_{i}(t-\tau)\right) .
\end{aligned}
$$

This delay is caused by the fact that the agent needs some memory to store the outdated information from its neighboring agents. It found that this delay can induce quasiconsensus; that is, all agents have the same velocity but keep a distance from each other eventually. Therefore, the delay in this sense looks very interesting. This paper considers quasi-consensus of high-order multi-agent systems with the delay in the sense of memory effect. Based on the Nyquist stability criterion, some results on the quasi-consensus of high-order multi-agent systems are obtained. It is worth noting that the results on the consensus of high-order multiagent systems with a protocol of communication delay hold similarly. Furthermore, some simulations are given to verify the theoretical results.

Notations. Let $\mathbb{R}^{n}$ denote the $n$-dimensional Euclidian space. Let $0_{N}$ and $I_{N}$ be the $N \times N$ zero matrix and identity matrix, respectively. If $M$ is a matrix, then $\operatorname{rank} M$ and $\sigma(M)$ denote the rank and the spectrum of $M$, respectively. Let $\mathbf{1}$ and $\mathbf{0}$ denote the $N \times 1$ column vectors of all ones and all zeros, respectively. The symbol $\otimes$ denotes the Kronecker product.

\section{Preliminaries and Model}

Consider a multi-agent systems of $N$ agents. The dynamics of each agent is governed by the following general $\ell$ th-order integrator:

$$
\begin{gathered}
\dot{\xi}_{i}^{(0)}=\xi_{i}^{(1)}, \\
\vdots \\
\dot{\xi}_{i}^{(\ell-2)}=\xi_{i}^{(\ell-1)}, \\
\dot{\xi}_{i}^{(\ell-1)}=u_{i},
\end{gathered}
$$

where $i=1,2, \ldots, N, \ell \geq 1$ is a positive integer and denotes the order of the differential equations, $\xi_{i}^{(k)} \in \mathbb{R}^{m}, k=$ $0,1, \ldots, \ell-1$, are the states of agent $i, u_{i} \in \mathbb{R}^{m}$ is the consensus protocol, and $\xi_{i}^{(k)}$ denotes the $k$ th-order derivative of $\xi_{i}$. The initial state denotes $\xi_{i 0}=\left[\xi_{i}(0), \xi_{i}^{(1)}(0), \ldots, \xi_{i}^{(\ell-1)}(0)\right]$.

We use the weighted undirected graph $\mathscr{G}$ to model the interaction topology of the multi-agent systems; that is, each vertex represents an agent of systems; each arc (edge) $e_{j i}$ represents that there is a communication link from agent $j$ to agent $i . a_{i j}>0$ is the weight of the communication link $e_{j i}$. One can see [26] for more details. Now, we propose the following consensus protocol:

$$
\begin{aligned}
u_{i}= & -\sum_{k \in M_{1}} c_{k} \xi_{i}^{(k)} \\
& -\alpha \sum_{j=1}^{N} a_{i j}\left(\sum_{k \in M_{2}} \gamma_{k}\left(\xi_{i}^{(k)}-\xi_{j}^{(k)}\right)\right),
\end{aligned}
$$

where $M=\{0,1,2, \ldots, \ell-1\}$. Let $M_{1}, M_{2} \subset M$ be two nonempty subsets such that $M_{1} \cup M_{2}=M, M_{1} \cap M_{2}=\emptyset$, $\alpha$ is the coupling strength and $\gamma_{k}>0$ is the feedback gain of relative information. $c_{k} \neq 0 \Leftrightarrow k \in M_{1}$, otherwise, $c_{k}=0$ and let $c_{0}=0 ; \gamma_{k} \neq 0 \Leftrightarrow k \in M_{2}$, otherwise, $\gamma_{k}=0$ and let $\gamma_{0} \neq 0$. Let $n_{0}=\min \left\{k: k \in M_{1}\right\}$.

Let $\xi^{(0)}=\left[\xi_{1}^{(0)^{T}}, \xi_{2}^{(0)^{T}}, \ldots, \xi_{N}^{(0)^{T}}\right]^{T}$ and $\xi^{(n+1)}=\dot{\xi}^{(n)}, n=$ $0,1, \ldots, \ell-2$. By employing the consensus protocol (3), the dynamics equations of all agents can be written as follows:

$$
\left[\begin{array}{c}
\dot{\xi}^{(0)} \\
\dot{\xi}^{(1)} \\
\vdots \\
\dot{\xi}^{(\ell-1)}
\end{array}\right]=\left(\Phi \otimes I_{m}\right)\left[\begin{array}{c}
\xi^{(0)} \\
\xi^{(1)} \\
\vdots \\
\xi^{(\ell-1)}
\end{array}\right]
$$


where

$$
\begin{gathered}
\Phi=A \otimes I_{N}-\alpha B \otimes L, \\
A=\left[\begin{array}{ccccc}
0 & 1 & 0 & \cdots & 0 \\
0 & 0 & 1 & \cdots & 0 \\
\vdots & \vdots & \vdots & \vdots & \vdots \\
0 & 0 & 0 & \cdots & 1 \\
0 & -c_{1} & -c_{2} & \cdots & -c_{\ell-1}
\end{array}\right]_{\ell \times \ell}, \\
B=\left[\begin{array}{cccc}
0 & 0 & \cdots & 0 \\
0 & 0 & \cdots & 0 \\
\vdots & \vdots & \vdots & \vdots \\
0 & 0 & \cdots & 0 \\
\gamma_{0} & \gamma_{1} & \cdots & \gamma_{\ell-1}
\end{array}\right]_{\ell \times \ell},
\end{gathered}
$$

$L=\left[\ell_{i j}\right]$ is the associated Laplacian of $\mathscr{G}$; that is $\ell_{i i}=\sum_{j \neq i} a_{i j}$, $\ell_{i j}=-a_{i j}$. It is easy to note that

$$
\Phi=\left[\begin{array}{ccccc}
0_{N} & I_{N} & 0_{N} & \cdots & 0_{N} \\
0_{N} & 0_{N} & I_{N} & \cdots & 0_{N} \\
\vdots & \vdots & \vdots & \vdots & \vdots \\
0_{N} & 0_{N} & 0_{N} & \cdots & I_{N} \\
-\alpha \gamma_{0} L & -\Delta_{1} & -\Delta_{2} & \cdots & -\Delta_{\ell-1}
\end{array}\right],
$$

where $\Delta_{k}=c_{k} I_{N}+\alpha \gamma_{k} L$.

Definition 1. The multi-agent systems governed by (4) are said to achieve consensus if $\lim _{t \rightarrow \infty}\left\|\xi_{i}^{(k)}(t)-\xi_{j}^{(k)}(t)\right\|=0$, $k=0,1,2, \ldots, \ell-1, i, j=1,2, \ldots, N, i \neq j$, for any initial values.

Remark 2. For convenience, we assume $m=1$ in the following discussion; however, all the results hereafter remain valid for $m>1$ by the Kronecker product.

\section{Consensus Analysis}

In this section, we consider the consensus problem defined in the Section 2 for the multi-agent systems (4) with fixed topology. To this end, we establish the following lemmas which are needed for the main results.

Denote $\Gamma=I_{N} \otimes A-\alpha L \otimes B$. One has the following lemmas.

Lemma 3. $\Phi$ is similar to $\Gamma$.

Proof. See the appendix.

Lemma 4. Assume that $\Phi$ is defined in (5) and $\lambda_{1}, \lambda_{2}, \ldots$, $\lambda_{N} \in \sigma(L)$; then the characteristic polynomial of $\Phi$ is

$$
\begin{aligned}
\operatorname{det}\left(\mu I_{N \ell}-\Phi\right)=\prod_{i=1}^{N}\left(\mu^{\ell}+\delta_{\ell-1}^{i} \mu^{\ell-1}\right. & \\
& \left.+\cdots+\delta_{1}^{i} \mu+\alpha \lambda_{i} \gamma_{0}\right),
\end{aligned}
$$

where $\delta_{k}^{i}=c_{k}+\alpha \lambda_{i} \gamma_{k}, k=1,2, \ldots, \ell-1, i=1,2, \ldots, N$.

Proof. See the appendix.
Lemma 5. $\Phi$ has at least $n_{0}$ zero eigenvalues. It has exactly $n_{0}$ zero eigenvalues if and only if $L$ has a simple zero eigenvalue. If $L$ has a simple zero eigenvalue, then the geometric multiplicity of the zero eigenvalue of $\Phi$ equals one. Moreover, the left and right eigenvector of $\Phi$ associated with eigenvalue zero are as follows:

$$
\begin{gathered}
v_{l}=\frac{1}{c_{n_{0}}}\left[c_{1}, \ldots, c_{\ell-1}, 1\right] \otimes \mathbf{1}^{T}, \\
w_{r}=\frac{1}{\sqrt{N}} e_{1} \otimes \mathbf{1} .
\end{gathered}
$$

Proof. See the appendix.

Based on the previous lemmas, here we give the main result.

Theorem 6. Under the consensus protocol (3), the multi-agent systems (4) achieve the consensus if and only if $\Phi$ has exactly $n_{0}$ zero eigenvalues and all of other eigenvalues are negative.

Proof (Sufficiency). If $\Phi$ has exactly $n_{0}$ zero eigenvalues, then $\Phi$ has the Jordan canonical form $\left[\begin{array}{ll}J_{1} & \\ & J_{2}\end{array}\right]$, where

$$
J_{1}=\left[\begin{array}{ccccc}
0 & 1 & 0 & \cdots & 0 \\
0 & 0 & 1 & \cdots & 0 \\
\vdots & \vdots & \vdots & \vdots & \vdots \\
0 & 0 & 0 & \cdots & 1 \\
0 & 0 & 0 & \cdots & 0
\end{array}\right]_{n_{0} \times n_{0}}
$$

$J_{2}$ is the upper diagonal block matrix with diagonal entries being the nonzero eigenvalues of $\Phi$. Moreover, one has

$$
\begin{aligned}
\Phi= & {\left[w_{1}, w_{2}, \ldots, w_{\ell N}\right] } \\
& \times\left[\begin{array}{cc}
J_{1} & 0_{n_{0} \times\left(N \ell-n_{0}\right)} \\
0_{\left(N \ell-n_{0}\right) \times n_{0}} & J_{2}
\end{array}\right] \\
& \times\left[\begin{array}{c}
v_{1}^{T} \\
v_{2}^{T} \\
\vdots \\
v_{\ell N}^{T}
\end{array}\right]
\end{aligned}
$$

where $w_{i} \in \mathbb{R}^{\ell N}, i=1,2, \ldots, \ell N$, can be chosen to be the right eigenvectors or generalized eigenvectors of $\Phi, v_{i} \in$ $\mathbb{R}^{\ell N}, i=1,2, \ldots, \ell N$, can be chosen to be the left eigenvectors or generalized eigenvectors of $\Phi$. Without of loss generality, assume that $M_{2}=\left\{0,1, \ldots, n_{0}-1\right\}$ and $M_{1}=$ $\left\{n_{0}, n_{0}+1, \ldots, \ell-1\right\}$. Let $w_{1}=\left[\mathbf{1}^{T}, \mathbf{0}^{T}, \ldots, \mathbf{0}^{T}\right]^{T}, w_{2}=$ $\left[\mathbf{0}^{T}, \mathbf{1}^{T}, \ldots, \mathbf{0}^{T}\right]^{T}, \ldots, w_{n_{0}}=[\underbrace{\mathbf{0}^{T}, \mathbf{0}^{T}, \ldots, \mathbf{1}^{T}}_{n_{0}}, \underbrace{\mathbf{0}^{T}, \ldots, \mathbf{0}^{T}}_{\ell-n_{0}}]^{T}$; one can verify that $w_{1}, w_{2}, \ldots, w_{n_{0}}$ are a right eigenvector and $n_{0}-1$ generalized right eigenvectors of $\Phi$ associated with zero eigenvalue, respectively. Since $\Phi$ has exactly $n_{0}$ zero eigenvalues, without loss of generality, one can denote them by $\mu_{1}=$ $\mu_{2}=\cdots=\mu_{n_{0}}=0$. By Lemma 5 , we know that $L$ has a simple 
zero eigenvalue, which implies that there is a nonnegative vector $p=(1 / N) \mathbf{1}$ such that $p^{T} L=\mathbf{0}, p^{T} \mathbf{1}=1$ by [16]. Then we choose, $v_{1}=\left(1 / c_{n_{0}}\right)[c_{n_{0}} p^{T}, \ldots, c_{\ell-1} p^{T}, p^{T}, \underbrace{\mathbf{0}^{T}, \ldots, \mathbf{0}^{T}}_{n_{0}-1}]^{T}, \ldots$, $v_{2}=\left(1 / c_{n_{0}}\right)[\mathbf{0}^{T}, c_{n_{0}} p^{T}, \ldots, c_{\ell-1} p^{T}, p^{T}, \underbrace{\mathbf{0}^{T}, \ldots, \mathbf{0}^{T}}_{n_{0}-2}]^{T}, \ldots, v_{n_{0}}=$ $\left(1 / c_{n_{0}}\right)[\underbrace{\mathbf{0}^{T}, \ldots, \mathbf{0}^{T}}_{n_{0}-1}, c_{n_{0}} p^{T}, \ldots, c_{\ell-1} p^{T}, p^{T}]^{T}$. It follows from Lemma 5 that they are $n_{0}-1$ generalized left eigenvectors and a left eigenvector of $\Phi$ associated with eigenvalue zero, respectively. Moreover, $v_{i}^{T} w_{i}=1, i=1,2, \ldots, n_{0}$. It is noted that

$$
\begin{aligned}
e^{\Phi t}= & {\left[w_{1}, w_{2}, \ldots, w_{\ell N}\right] } \\
& \times\left[\begin{array}{cc}
e^{J_{1} t} & 0_{n_{0} \times\left(N \ell-n_{0}\right)} \\
0_{\left(N \ell-n_{0}\right) \times n_{0}} & e^{I_{2} t}
\end{array}\right]\left[\begin{array}{c}
v_{1}^{T} \\
v_{2}^{T} \\
\vdots \\
v_{\ell N}^{T}
\end{array}\right],
\end{aligned}
$$

where $\lim _{t \rightarrow \infty} e^{J_{2} t}=0_{N \ell-n_{0}}$ since the nonzero eigenvalues have negative real parts and

$$
e^{J_{1} t}=\left[\begin{array}{ccccc}
1 & t & \frac{t^{2}}{2 !} & \cdots & \frac{t^{n_{0}-1}}{\left(n_{0}-1\right) !} \\
0 & 1 & t & \cdots & \frac{t^{n_{0}-2}}{\left(n_{0}-2\right) !} \\
\vdots & \vdots & \vdots & \vdots & \vdots \\
0 & 0 & 0 & \cdots & t \\
0 & 0 & 0 & \cdots & 1
\end{array}\right]_{n_{0} \times n_{0}}
$$

Therefore, for enough large $t$, the dominant terms of $e^{\Phi t}$ denoted by $\Phi_{d}$ is

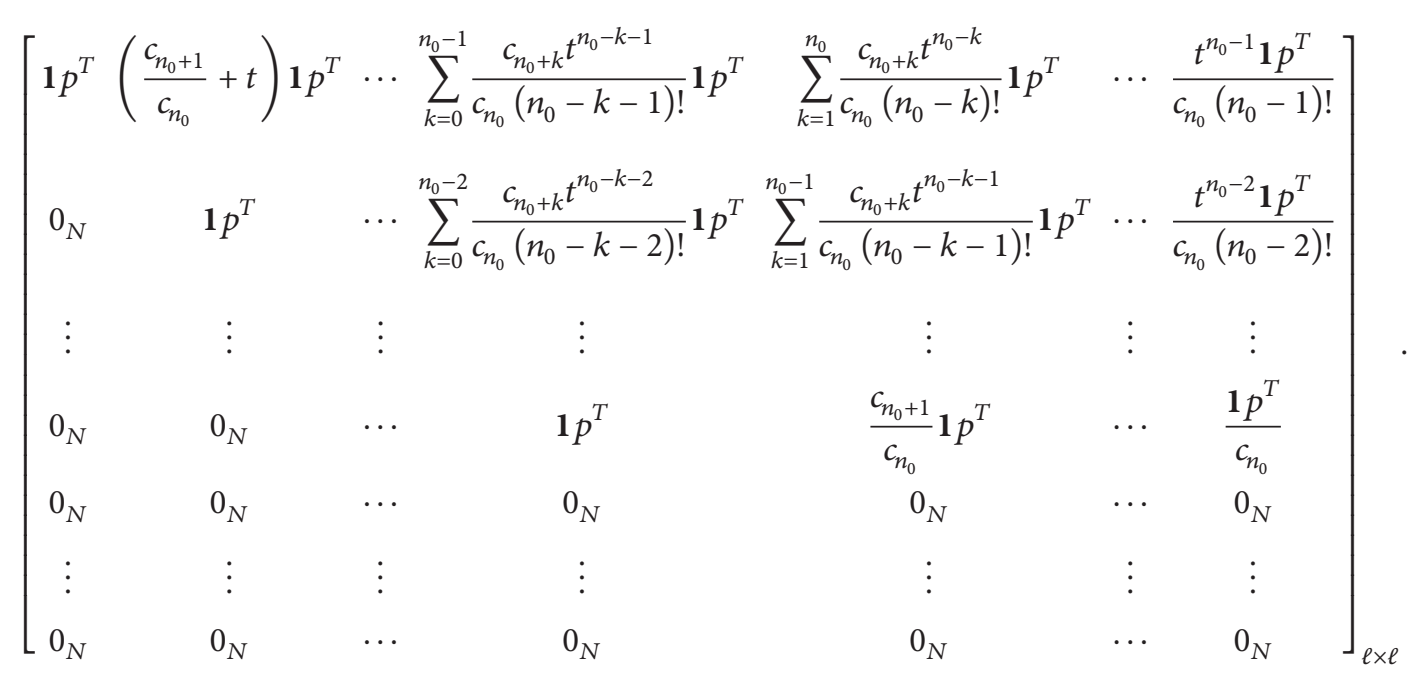

Thus, we write $\Phi_{d}$ by a compact form

$$
\left[\begin{array}{cccc}
\mathbf{1} p^{T} & r_{12}(t) \mathbf{1} p^{T} \cdots & r_{1 n_{0}}(t) \mathbf{1} p^{T} \cdots & r_{1 \ell}(t) \mathbf{1} p^{T} \\
0_{N} & \mathbf{1} p^{T} \cdots & r_{2 n_{0}}(t) \mathbf{1} p^{T} \cdots & r_{2 \ell}(t) \mathbf{1} p^{T} \\
\vdots & \vdots & \vdots & \vdots \\
0_{N} & 0_{N} \cdots & \mathbf{1} p^{T} \cdots & r_{n_{0} \ell}(t) \mathbf{1} p^{T} \\
0_{N} & 0_{N} \cdots & 0_{N} \cdots & 0_{N} \\
\vdots & \vdots & \vdots & \vdots \\
0_{N} & 0_{N} \cdots & 0_{N} \cdots & 0_{N}
\end{array}\right],
$$

where $r_{i j}(t), j>i$ is a polynomial of $t$ with degree $j-i$. It follows from (4) that

$$
\left[\begin{array}{c}
\xi^{(0)}(t) \\
\xi^{(1)}(t) \\
\vdots \\
\xi^{(\ell-1)}(t)
\end{array}\right]=e^{\Phi t}\left[\begin{array}{c}
\xi^{(0)}(0) \\
\xi^{(1)}(0) \\
\vdots \\
\xi^{(\ell-1)}(0)
\end{array}\right],
$$

that is, for enough large $t$,

$$
\begin{aligned}
\xi^{(k)}(t)= & {\left[\xi_{1}^{(k)}(t), \xi_{2}^{(k)}(t), \ldots, \xi_{N}^{(k)}(t)\right]^{T} } \\
= & \mathbf{1} p^{T} \xi^{(k)}(0)+r_{k+1, k+2}(t) \mathbf{1} p^{T} \xi^{(k+1)}(0) \\
& +\cdots+r_{k+1, \ell}(t) \mathbf{1} p^{T} \xi^{(\ell-1)}(0), \quad k \leq n_{0}-1,
\end{aligned}
$$

which implies that $\xi_{i}^{(k)}(t) \rightarrow \xi_{j}^{(k)}(t)$ as $t \rightarrow \infty$ for $k \leq n_{0}-1$. 
If $k \geq n_{0}$, then $\xi^{(k)}(t) \rightarrow \mathbf{0}$ as $t \rightarrow \infty$. Therefore, $\xi_{i}^{(k)}(t) \rightarrow \xi_{j}^{(k)}(t)$ as $t \rightarrow \infty$, for all $i \neq j, k=0,1, \ldots, \ell-1$. This implies that the multi-agent systems achieve the consensus.

(Necessity). By reduction to absurdity, suppose that the sufficient condition dose not hold; then there are two cases may be hold.

Case 1. $\Phi$ has more than $n_{0}$ zero eigenvalues.

Case 2. $\Phi$ has exactly $n_{0}$ zero eigenvalues, but it has at least one eigenvalue with nonnegative real part.

If Case 1 holds, then $\operatorname{rank}\left(\lim _{t \rightarrow \infty} e^{\Phi t}\right) \quad \geq$ $\operatorname{rank}\left(\lim _{t \rightarrow \infty} e^{J_{1} t}\right)>n_{0}$.

If Case 2 holds, then $\lim _{t \rightarrow \infty} e^{J_{2} t} \neq 0_{N \ell-n_{0}}$, and $\operatorname{rank}\left(\lim _{t \rightarrow \infty} e^{\Phi t}\right) \geq \operatorname{rank}\left(\lim _{t \rightarrow \infty} e^{J_{1} t}\right)+\operatorname{rank}\left(\lim _{t \rightarrow \infty} e^{J_{2} t}\right)>$ $n_{0}$. However, it follows from $\Phi_{d}$ (the dominant terms of $e^{\Phi t}$ ) that if the consensus is achieved, then $\operatorname{rank}\left(\lim _{t \rightarrow \infty} e^{\Phi t}\right) \leq n_{0}$. This is a contradiction.

Remark 7. From Theorem 6, if (4) achieves the consensus, then $\Phi$ has exactly $n_{0}$ zero eigenvalues. Therefore, $L$ has a simple zero eigenvalue, which implies that the information exchange topology $\mathscr{G}$ is connected.

Remark 8. Compared to the consensus protocols proposed in [16-19], protocol (3) only depends on the own partial information of agents and the partial relative states with its neighbors, which does not need the global information of agents or the global relative information. It is convenient to design when global information states cannot be available.

To illustrate the previous result, we consider the following example.

Example 9. Considering the following third-order system of 8 agents, where the dynamics of each agent are governed by

$$
\dot{x}_{i}=v_{i}, \quad \dot{v}_{i}=a_{i}, \quad \dot{a}_{i}=u_{i},
$$

where $x_{i}, v_{i}, a_{i}, i=1,2, \ldots, 8$, denote the position, velocity, and acceleration of agent $i$, respectively, let Laplacian matrix $L$ be

$$
\left[\begin{array}{cccccccc}
1 & 0 & -1 & 0 & 0 & 0 & 0 & 0 \\
0 & 2 & -1 & 0 & 0 & -1 & 0 & 0 \\
-1 & -1 & 4 & -1 & 0 & 0 & -1 & 0 \\
0 & 0 & -1 & 2 & -1 & 0 & 0 & 0 \\
0 & 0 & 0 & -1 & 1 & 0 & 0 & 0 \\
0 & -1 & 0 & 0 & 0 & 1 & 0 & 0 \\
0 & 0 & -1 & 0 & 0 & 1 & 0 & 0 \\
0 & 0 & 0 & 0 & 0 & 0 & -1 & 1
\end{array}\right] .
$$

It follows from the $L$ that the graph is connected. Moreover, the eigenvalues of $L$ are $0,0.382,0.6086,1,1,2.2271,2.618$, and 5.1642. If we choose $\alpha=1, \gamma_{0}=1, \gamma_{1}=2$, and $c_{2}=2$, then $n_{0}=2$. Furthermore, $\Phi$ has 2 zero eigenvalues and the real parts of all other eigenvalues are negative. Let the initial values be the $x(0)=[10,20,30,5,-10,-20,-30,-5], v(0)=$

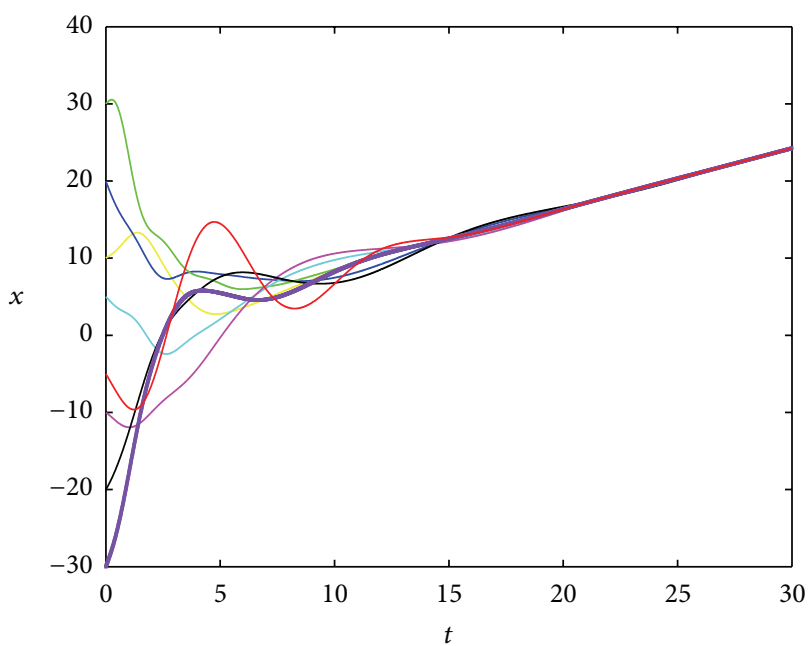

FIgURE 1: The trajectories of $x_{i}, i=1,2, \ldots, 8$.

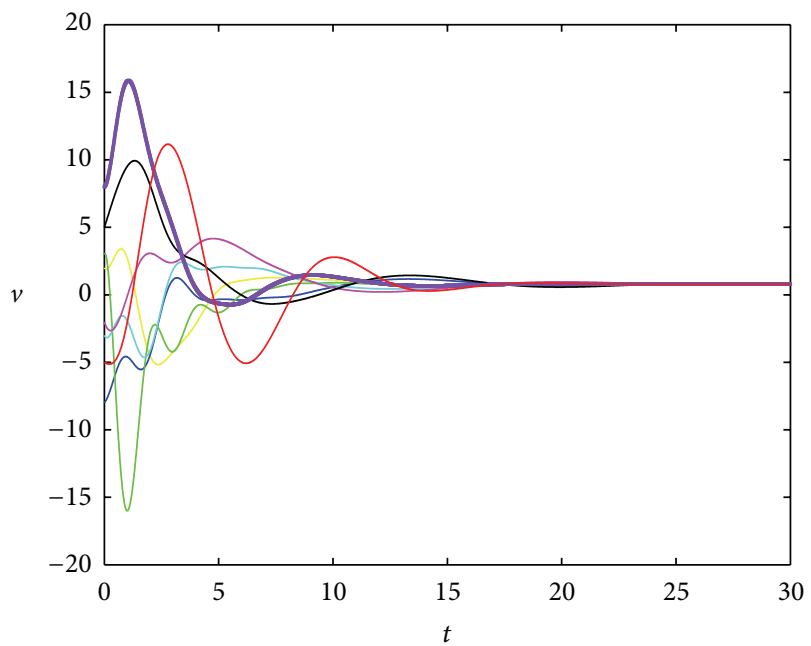

FIGURE 2: The trajectories of $v_{i}, i=1,2, \ldots, 8$.

$[2,-8,3,-3,-2,5,8,-5], a(0)=[-2,2,3,-3,5,-5,1,-1]$. By Theorem 6 , consensus in dynamical multi-agent systems (18) with network topology (19) can be achieved. Figures 1, 2, and 3 show the plots of $x_{i}, v_{i}, a_{i}, i=1,2, \ldots, 8$, respectively.

\section{Quasi-Consensus Problem with Delays}

Because of the limited transmission speed or memory effect, there exist nonnegligible delays in the communication process. Therefore, it is necessary to consider the influence of delays on the consensus. In [25], the authors considered the delays in the sense of memory store by the fact that the agent uses the memory to store the outdated information of its neighbors. Such delays are so interesting that can deduce quasi-consensus. In this section, we consider the consensus 


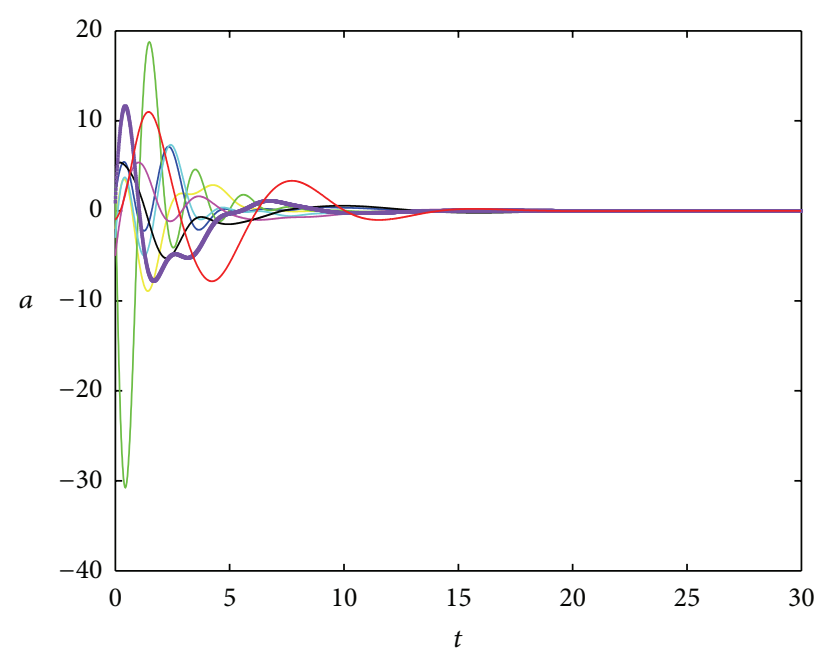

FIgURE 3: The trajectories of $a_{i}, i=1,2, \ldots, 8$.

problem with the protocol of delays in the sense of memory store as follows:

$$
\begin{aligned}
u_{i}= & -\sum_{k \in M_{1}} c_{k} \xi_{i}^{(k)}+\alpha \sum_{j=1}^{N} a_{i j} \sum_{k \in M_{2}} \gamma_{k}\left(\xi_{j}^{(k)}(t)-\xi_{i}^{(k)}(t)\right) \\
& +\sum_{k \in M_{1}} c_{k} \xi_{i}^{(k)}(t-\tau) \\
& -\alpha \sum_{j=1}^{N} a_{i j} \sum_{k \in M_{2}} \gamma_{k}\left(\xi_{j}^{(k)}(t-\tau)-\xi_{i}^{(k)}(t-\tau)\right),
\end{aligned}
$$

where $\tau>0$. Let $\eta_{i}=\left[\xi_{i}^{(0)}, \xi_{i}^{(1)}, \ldots, \xi_{i}^{(\ell-1)}\right], \eta=\left[\eta_{1}^{T}, \eta_{2}^{T}\right.$, $\left.\ldots, \eta_{N}^{T}\right]^{T}$.

With the consensus protocol (20), the closed loop of multi-agent systems (2) can be written as

$$
\begin{aligned}
\dot{\eta}_{i}= & A \eta_{i}-\widetilde{A} \eta_{i}(t-\tau)-\alpha \sum_{j=1}^{N} l_{i j} B \eta_{j} \\
& +\alpha \sum_{j=1}^{N} l_{i j} B \eta_{j}(t-\tau),
\end{aligned}
$$

where $A, B$ are defined as (5) and

$$
\widetilde{A}=\left[\begin{array}{cccc}
0 & 0 & \cdots & 0 \\
0 & 0 & \cdots & 0 \\
\vdots & \vdots & \vdots & \vdots \\
0 & 0 & \cdots & 0 \\
0 & -c_{1} & \cdots & -c_{\ell-1}
\end{array}\right]_{\ell \times \ell}
$$

Therefore,

$$
\begin{aligned}
\dot{\eta}= & \left(I_{N} \otimes A\right) \eta-\left(I_{N} \otimes \widetilde{A}\right) \eta(t-\tau) \\
& -\alpha(L \otimes B) \eta+\alpha(L \otimes B) \eta(t-\tau) .
\end{aligned}
$$

Let $J$ be the Jordan matrix associate with the $L$. Therefore, there exists orthogonal matrix $Q$ such that $Q^{T} L Q=J=$ $\operatorname{diag}\left\{\lambda_{1}, \lambda_{2}, \ldots, \lambda_{N}\right\}$. Then,

$$
\begin{aligned}
\left(Q^{T} \otimes I_{\ell}\right) \dot{\eta}(t) \\
\quad=\left(Q^{T} \otimes I_{\ell}\right)\left[\left(I_{N} \otimes A\right)-\alpha(L \otimes B)\right] \eta(t) \\
\quad-\left(Q^{T} \otimes I_{\ell}\right)\left[\left(I_{N} \otimes \widetilde{A}\right)-\alpha(L \otimes B)\right] \eta(t-\tau) \\
\quad=\left[\left(I_{N} \otimes A\right)-\alpha(J \otimes B)\right]\left(Q^{T} \otimes I_{\ell}\right) \eta(t) \\
-\left[\left(I_{N} \otimes \widetilde{A}\right)-\alpha(J \otimes B)\right]\left(Q^{T} \otimes I_{\ell}\right) \eta(t-\tau) .
\end{aligned}
$$

Let $\theta(t)=\left(Q^{T} \otimes I_{\ell}\right) \eta(t)=\left[\theta_{1}^{T}(t), \theta_{2}^{T}(t), \ldots, \theta_{N}^{T}(t)\right]^{T}$. Then, it follows from (24) that

$$
\begin{aligned}
\dot{\theta}(t)= & {\left[\left(I_{N} \otimes A\right)-\alpha(J \otimes B)\right] \theta(t) } \\
& -\left[\left(I_{N} \otimes \widetilde{A}\right)-\alpha(J \otimes B)\right] \theta(t-\tau) ;
\end{aligned}
$$

that is

$$
\begin{aligned}
\dot{\theta}_{i}(t)= & \left(A-\alpha \lambda_{i} B\right) \theta_{i}(t) \\
& -\left(\widetilde{A}-\alpha \lambda_{i} B\right) \theta_{i}(t-\tau) .
\end{aligned}
$$

The characteristic equation of the multi-agent system (26) is

$$
\begin{aligned}
& \lambda^{\ell}+\left[c_{\ell-1}+\alpha \lambda_{i} \gamma_{\ell-1}\right]\left(1-e^{-\lambda \tau}\right) \lambda^{\ell-1} \\
& \quad+\cdots+\left[c_{1}+\alpha \lambda_{i} \gamma_{1}\right]\left(1-e^{-\lambda \tau}\right) \lambda \\
& \quad+\alpha \lambda_{i} \gamma_{0}\left(1-e^{-\lambda \tau}\right)=0, \quad i=1,2, \ldots, N
\end{aligned}
$$

Definition 10. The multi-agent systems (2) are said to achieve quasi-consensus if

$$
\begin{aligned}
& \lim _{t \rightarrow \infty}\left\|\xi_{i}^{(0)}(t)-\xi_{j}^{(0)}(t)\right\|=c_{i j} \\
& \lim _{t \rightarrow \infty}\left\|\xi_{i}^{(k)}(t)-\xi_{j}^{(k)}(t)\right\|=0
\end{aligned}
$$

for any initial conditions, where $c_{i j}$ are constants, for all $k=$ $1,2, \ldots, \ell-1$ and $i, j=1,2, \ldots, N$.

Theorem 11. Assume that $\mathscr{G}$ is connected. Quasi-consensus of multi-agent systems (2) with protocol (20) can be achieved if and only if each equation of $(27)(i=2,3, \ldots, N)$ has only simple zero root and the real parts of all other roots are negative.

Proof(Sufficiency). If $\mathscr{G}$ is connected, then $L$ has only a simple zero eigenvalue with associated right eigenvector $q_{1}=$ $(1 / \sqrt{N}) 1$, where $Q^{T} L Q=J, Q=\left[q_{1}, q_{2}, \ldots, q_{N}\right], q_{i}=$ $\left[q_{i 1}, q_{i 2}, \ldots, q_{i N}\right]^{T}, i=1,2, \ldots, N$. If each equation of $(27)$ $(i=2,3, \ldots, N)$ has only simple zero root and the real parts of all other roots are negative, then the states in (26) converge to some constants. It follows from the $\theta(t)=\left(Q^{T} \otimes I_{\ell}\right) \eta(t)$ 
that $\theta_{i}(t)=\left(q_{i}^{T} \otimes I_{\ell}\right) \eta(t)=\sum_{j=1}^{N} q_{i j} \eta_{j}(t)$. Denote $\theta_{i}(t)=$ $\left[\theta_{i 0}(t), \theta_{i 1}(t), \ldots, \theta_{i \ell-1}(t)\right]^{T}$; then one has

$$
\theta_{i k}(t)=\sum_{j=1}^{N} q_{i j} \xi_{j}^{(k)}(t), \quad k=0,1, \ldots, \ell-1 .
$$

Noting that $\dot{\theta}_{i k}(t)=\theta_{i k+1}(t)$, if one of the $\theta_{i 1}(t), \theta_{i 2}(t)$, $\ldots, \theta_{i \ell-1}(t)$ does not converge to zero, then $\lim _{t \rightarrow \infty}\left\|\theta_{i 0}(t)\right\|=$ $\infty$. Therefore, $\lim _{t \rightarrow \infty}\left\|\theta_{i k}(t)\right\|=0, i=2,3, \ldots, N, k=$ $1, \ldots, \ell-1$. Moreover, one has $\eta_{i}(t)=\sum_{j=1}^{N} q_{j i} \theta_{j}(t)$; therefore,

$$
\xi_{i}^{(k)}(t)=\sum_{j=1}^{N} q_{j i} \theta_{j k}(t), \quad k=0,1, \ldots, \ell-1 .
$$

Thus,

$$
\lim _{t \rightarrow \infty}\left\|\xi^{(k)}(t)-\frac{1}{\sqrt{N}}\left[\theta_{1 k}(t), \theta_{1 k}(t), \ldots, \theta_{1 k}(t)\right]^{T}\right\|=0
$$

where $k=1,2, \ldots, \ell-1, \dot{\theta}_{1}(t)=A \theta_{1}(t)$. Therefore, the quasiconsensus is reached.

(Necessity). If the quasi-consensus can be reached, then there exists $\sigma(t)=\left[\sigma_{0}(t), \sigma_{1}(t), \ldots, \sigma_{\ell-1}(t)\right]^{T}$ such that $\lim _{t \rightarrow \infty}\left\|\xi^{(k)}(t)-1_{N} \otimes \sigma_{k}(t)\right\|=0$. Since $G$ is connected, $L$ has only one zero eigenvalue with associated eigenvector $q_{1}=$ $(1 / \sqrt{N})$ 1. Thus, $\mathbf{0}=Q^{T} L \mathbf{1}=J Q^{T} \mathbf{1}=\left[\lambda_{1} q_{1}^{T} \mathbf{1}, \lambda_{2} q_{2}^{T} \mathbf{1}, \ldots\right.$, $\left.\lambda_{N} q_{N}^{T} \mathbf{1}\right]^{T}$; that is, $q_{i}^{T} \mathbf{1}=0, i=2,3, \ldots, N$. Therefore, it follows from $\theta_{i}(t)=q_{i 1} \eta_{1}(t)+q_{i 2} \eta_{2}(t)+\cdots+q_{i N} \eta_{N}(t)=$ $\left(q_{i}^{T} \otimes I_{\ell}\right) \eta(t)$ that

$$
\lim _{t \rightarrow \infty}\left\|\theta_{i k}(t)\right\|=\lim _{t \rightarrow \infty}\left\|\left(q_{i}^{T} \mathbf{1}\right) \otimes \sigma_{k}(t)\right\|=0,
$$

$i=2,3, \ldots, N, k=1,2, \ldots, \ell-1, \dot{\theta}_{1}(t)=A \theta_{1}(t)$. By way of contradiction, assume that the sufficient condition does not hold; then there are two cases.

Case 1. Each of $N-1$ equations $(27)(i=2,3, \ldots, N)$ has more than one zero roots.

Case 2. Each of $N-1$ equations (27) $(i=2,3, \ldots, N)$ has simple zero root; however, there exists nonnegative real part.

If one of the previous two cases holds, then there exists a $1 \leq k \leq \ell-1$ such that $\lim _{t \rightarrow \infty}\left\|\theta_{i k}(t)\right\| \neq 0$ for $i=2,3, \ldots, N$, which contradicts formula (32).

$e^{-\lambda \tau}$ can be expressed by Taylor expansion; therefore, (27) can be rewritten as follows:

$$
\lambda^{\ell}+\lambda\left[\tau-\sum_{k=2}^{\infty} \frac{(-\tau)^{k} \lambda^{k-1}}{k !}\right]_{k=0}^{\ell-1} \delta_{k}^{i} \lambda^{k}=0 .
$$

Since $\tau>0$ and $\delta_{0}^{i}=\alpha \lambda_{i} \gamma_{0}>0, i=2,3, \ldots, N$, (33) has only simple zero root for $i=2,3, \ldots, N$. When $s \neq 0$, let $F_{i}(\lambda)=$ $1+G_{i}(\lambda)$, where

$$
G_{i}(\lambda)=\frac{\sum_{k=0}^{\ell-1} \delta_{k}^{i} \lambda^{k}}{\lambda^{\ell}}\left(1-e^{-\lambda \tau}\right), \quad i=2,3, \ldots, N
$$

Based on the Nyquist stability criterion, therefore, the real parts of nonzero roots of (27) are negative if and only if $G_{i}(j \omega)$ does not enclose the point $(-1, j 0)$ for $\omega \in \mathbb{R}$, where $j$ is imaginary unit.

Without loss of generality, assume that $\ell$ is an odd number. Denote $S_{1}=\{0,2, \ldots, \ell-1\}$ and $S_{2}=\{1,3, \ldots, \ell-2\}$. By some calculations, one can obtain

$$
\begin{aligned}
G_{i}(j \omega)= & \frac{\sum_{k=0}^{\ell-1} \delta_{k}^{i}(j \omega)^{k}}{(j \omega)^{\ell}}\left(1-e^{-j \omega \tau}\right) \\
= & \frac{\sum_{k=0}^{\ell-1} \delta_{k}^{i} \omega^{k} j^{k+\ell}}{(-\omega)^{\ell}} 2 \sin \frac{\omega \tau}{2} e^{j((\pi-\omega \tau) / 2)} \\
= & \left(\left(j \sum_{k \in S_{1}} \delta_{k}^{i} \omega^{k}(-1)^{(k+\ell-1) / 2}\right.\right. \\
& \left.+\sum_{k \in S_{2}} \delta_{k}^{i} \omega^{k}(-1)^{(k+\ell) / 2}\right) \\
& \left.\times\left(-\omega^{\ell}\right)^{-1}\right) 2 \sin \frac{\omega \tau}{2} e^{j((\pi-\omega \tau) / 2)} .
\end{aligned}
$$

Let $a_{i}=\sum_{k \in S_{2}} \delta_{k}^{i} \omega^{k}(-1)^{(k+\ell) / 2}, \quad b_{i}=$ $\sum_{k \in S_{1}} \delta_{k}^{i} \omega^{k}(-1)^{(k+\ell-1) / 2}$.

Therefore,

$$
\begin{aligned}
G_{i}(j \omega) & =\frac{a_{i}+b_{i} j}{-\omega^{\ell}} 2 \sin \frac{\omega \tau}{2} e^{j((\pi-\omega \tau) / 2)} \\
& =\frac{2 \sin (\omega \tau / 2) \sqrt{a_{i}^{2}+b_{i}^{2}}}{\omega^{\ell}} e^{j\left(((-\pi-\omega \tau) / 2)+\arctan \left(b_{i} / a_{i}\right)\right)} .
\end{aligned}
$$

Therefore, Theorem 11 is equivalent to the following theorem

Theorem 12. Assume that $\mathscr{G}$ is connected. Quasi-consensus of multi-agent systems (2) with protocol (20) can be achieved if and only if

$$
G_{i}(j \omega)=\frac{2 \sin (\omega \tau / 2) \sqrt{a_{i}^{2}+b_{i}^{2}}}{\omega^{\ell}} e^{j\left(((-\pi-\omega \tau) / 2)+\arctan \left(b_{i} / a_{i}\right)\right)}
$$

does not enclose the point $(-1, j 0)$ for $i \in\{2,3, \ldots, N\}$ and $\omega \in \mathbb{R}$, where $a_{i}=\sum_{k \in S_{2}} \delta_{k}^{i} \omega^{k}(-1)^{(k+\ell) / 2}, b_{i}=$ $\sum_{k \in S_{1}} \delta_{k}^{i} \omega^{k}(-1)^{(k+\ell-1) / 2}$.

As the symmetric property of the Nyquist plot, we only need to consider the case of $\omega>0$. When $\omega \in(0,+\infty)$, 
$\left|G_{i}(j \omega)\right| \leq\left(2 \sum_{k=0}^{\ell-1} \delta_{k}^{i} \omega^{k}\right) / \omega^{\ell}$ is monotonously decreasing; furthermore,

$$
\begin{aligned}
& \arg \left(G_{i}(j \omega)\right) \\
& = \begin{cases}\arctan \frac{b_{i}}{a_{i}}-\frac{\pi}{2}-\frac{\omega \tau}{2}, & 2 n \pi \leq \frac{\omega \tau}{2}<2 n \pi+\pi ; \\
\arctan \frac{b_{i}}{a_{i}}+\frac{\pi}{2}-\frac{\omega \tau}{2}, & 2 n \pi+\pi \leq \frac{\omega \tau}{2}<2 n \pi+2 \pi,\end{cases}
\end{aligned}
$$

where $n \geq 0$ is the nonnegative integer.

When $\omega \in(0,+\infty), G_{i}(j \omega)$ crosses the negative real axis for the first time at $\omega_{1}^{i}$, one has

$$
\begin{gathered}
\frac{\omega_{1}^{i} \tau}{2}=\arctan \frac{b_{i}^{1}}{a_{i}^{1}}+\frac{\pi}{2}, \\
\left|G_{i}\left(j \omega_{1}^{i}\right)\right| \leq \frac{2 \sum_{k \in S_{2}} \delta_{k}^{i}\left(\omega_{1}^{i}\right)^{k}}{\left(\omega_{1}^{i}\right)^{\ell}},
\end{gathered}
$$

where $a_{i}^{1}=\sum_{k \in S_{2}} \delta_{k}^{i}\left(\omega_{1}^{i}\right)^{k}(-1)^{(k+\ell) / 2}, b_{i}^{1}=$ $\sum_{k \in S_{1}} \delta_{k}^{i}\left(\omega_{1}^{i}\right)^{k}(-1)^{(k+\ell-1) / 2}$.

Therefore, one has the following result equivalently.

Theorem 13. Assume that $\mathscr{G}$ is connected. Quasi-consensus of multi-agent systems (2) with protocol (20) can be achieved if and only if

$$
2 \sum_{k \in S_{2}} \delta_{k}^{i}\left(\omega_{1}^{i}\right)^{k}<\left(\omega_{1}^{i}\right)^{\ell}, \quad \text { for } i=2,3, \ldots, N,
$$

where $\omega_{1}^{i}$ is defined by $\omega_{1}^{i} \tau / 2=\arctan \left(b_{i}^{1} / a_{i}^{1}\right)+\pi / 2$.

$$
\begin{aligned}
& \text { Denote } \delta_{\max }=\max _{i, k} \delta_{k}^{i} \text {. If all } \omega_{1}^{i} \text { satisfy } \\
& 2 \sum_{k \in S_{2}} \delta_{\max }\left(\omega_{1}^{i}\right)^{k} \leq\left(\omega_{1}^{i}\right)^{\ell}, \quad \text { for } i=2,3, \ldots, N,
\end{aligned}
$$

then Theorem 13 holds.

Remark 14. If one considers consensus protocol with communication delays as follows:

$$
\begin{aligned}
u_{i}= & -\sum_{k \in M_{1}} c_{k} \xi_{i}^{(k)}+\alpha \sum_{j=1}^{N} a_{i j} \sum_{k \in M_{2}} \gamma_{k}\left(\xi_{j}^{(k)}(t)-\xi_{i}^{(k)}(t)\right) \\
& -\sum_{k \in M_{1}} c_{k} \xi_{i}^{(k)}(t-\tau) \\
& +\alpha \sum_{j=1}^{N} a_{i j} \sum_{k \in M_{2}} \gamma_{k}\left(\xi_{j}^{(k)}(t-\tau)-\xi_{i}^{(k)}(t-\tau)\right),
\end{aligned}
$$

then the similar results can be obtained.

Example 15. Considering the multi-agent systems which is similar to example 1 , here we take (20) as the quasi-consensus

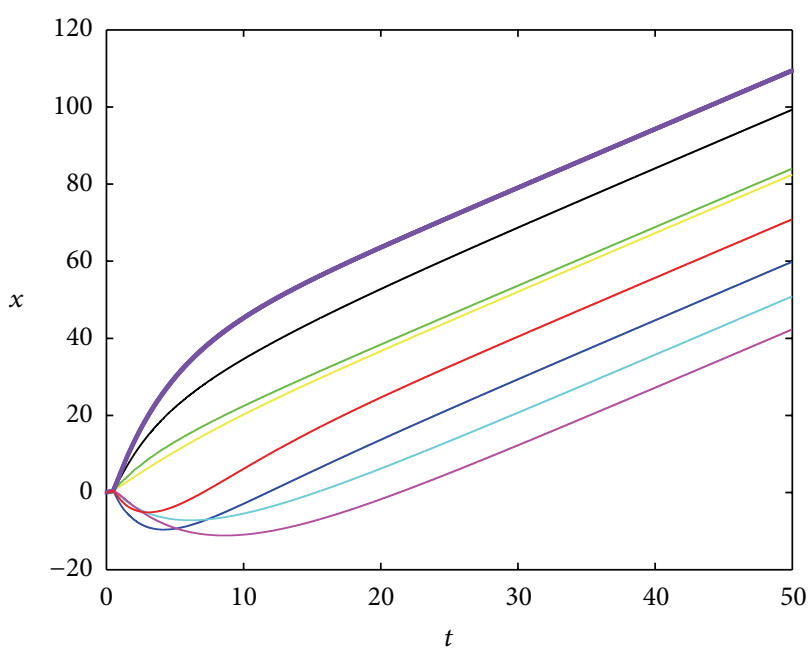

FIgURE 4: The trajectories of $x_{i}, i=1,2, \ldots, 8$.

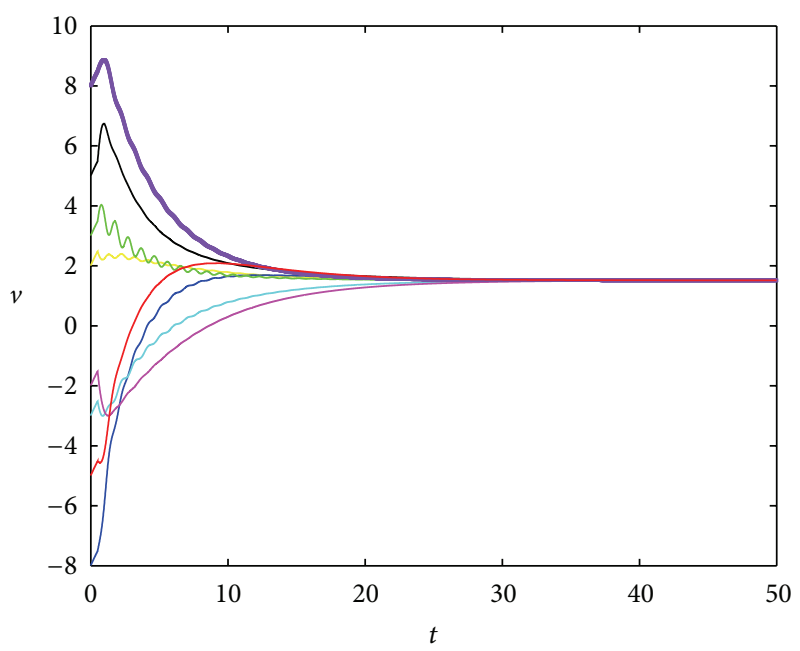

FIgURE 5: The trajectories of $v_{i}, i=1,2, \ldots, 8$.

protocol. We take the same parameters; that is, the Laplacian matrix $L$ is also

$$
\left[\begin{array}{cccccccc}
1 & 0 & -1 & 0 & 0 & 0 & 0 & 0 \\
0 & 2 & -1 & 0 & 0 & -1 & 0 & 0 \\
-1 & -1 & 4 & -1 & 0 & 0 & -1 & 0 \\
0 & 0 & -1 & 2 & -1 & 0 & 0 & 0 \\
0 & 0 & 0 & -1 & 1 & 0 & 0 & 0 \\
0 & -1 & 0 & 0 & 0 & 1 & 0 & 0 \\
0 & 0 & -1 & 0 & 0 & 1 & 0 & 0 \\
0 & 0 & 0 & 0 & 0 & 0 & -1 & 1
\end{array}\right],
$$

$\alpha=1, \gamma_{0}=1, \gamma_{1}=2$ and $c_{2}=2$ and $n_{0}=2$. Then, eigenvalues of $L$ are $\lambda_{1}=0, \lambda_{2}=0.382, \lambda_{3}=0.6086, \lambda_{4}=\lambda_{5}=1$, $\lambda_{6}=2.2271, \lambda_{7}=2.618$, and $\lambda_{8}=5.1642$. By simulation, $\tau_{\max }=1.3224$. Moreover, we choose $\tau=0.5$. By Theorem 13, one knows that the quasi-consensus can be reached. Figures 4, 5 and 6 show that the quasi-consensus is reached. 


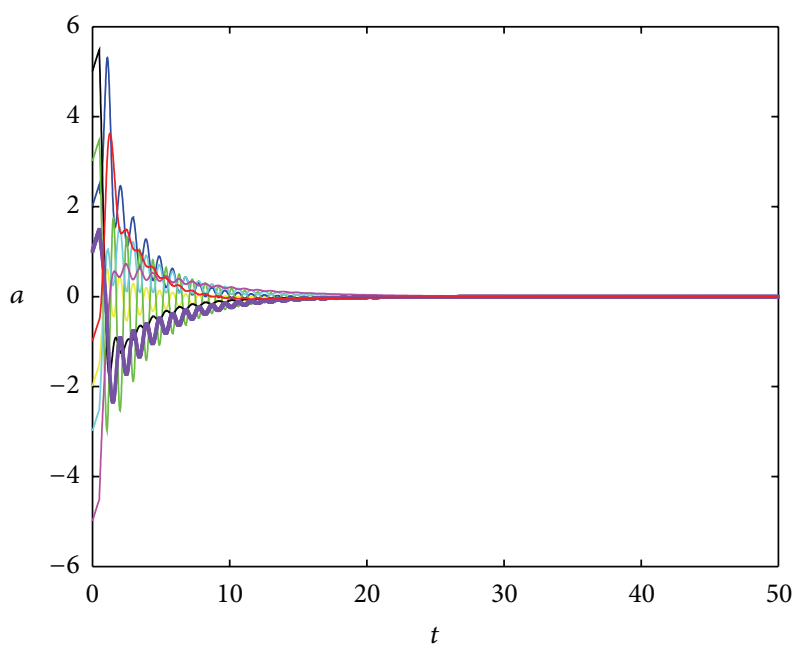

Figure 6: The trajectories of $a_{i}, i=1,2, \ldots, 8$.

\section{Conclusions}

When the global states of agents and global relative information are unavailable, this paper designs a consensus protocol which only depends on the own partial information of agents and partial relative information with its neighbors. Under the consensus protocol, a necessary and sufficient condition for guaranteeing the consensus for a high-order integrator multi-agent systems is established. Moreover, the consensus protocol shows that the consensus can be reached when the own states of agents are complementary to the relative information with its neighbors; the consensus may not be reached when there are only a few information exchanges. Moreover, the paper also considers the quasi-consensus problem under the protocol with the delays. It is interesting that multi-agent systems can reach consensus without delays and quasi-consensus with delays.

\section{Appendix}

Proof of Lemma 3. In fact, let

$$
x=\left[\left(\xi^{(0)}\right)^{T},\left(\xi^{(1)}\right)^{T}, \ldots,\left(\xi^{(\ell-1)}\right)^{T}\right]^{T}
$$

where $\xi=\left[\xi_{1}, \xi_{2}, \ldots, \xi_{N}\right]^{T}$. Then $\dot{x}=\Phi x$. On the other hand, let $\eta_{i}=\left[\xi_{i}^{(0)}, \xi_{i}^{(1)}, \ldots, \xi_{i}^{(\ell-1)}\right]^{T}$ and $y=\left[\eta_{1}^{T}, \eta_{2}^{T}, \ldots, \eta_{N}^{T}\right]^{T}$; then

$$
\dot{y}=\Gamma y \text {. }
$$

Noting that $y$ is a rearrange of $x$, there exists a nonsingular matrix $P$ in which every row and every column has only simple one such that $y=P x$, which together with (A.2)

$$
\dot{x}=P^{-1} \Gamma P x
$$

that is, $\Phi=P^{-1} \Gamma P$. Therefore, $\Phi$ is similar to $\Gamma$. Moreover, $\Gamma$ and $\Phi$ have the same characteristic polynomial and eigenvalues.
Proof of Lemma 4. Let $Q$ be a nonsingular matrix such that $Q^{-1} L Q=J$, where $J$ is the Jordan matrix associated to $L$. Thus

$$
\begin{aligned}
\operatorname{det}\left(\mu I_{N \ell}-\Phi\right) & =\operatorname{det}\left(\mu I_{N \ell}-\Gamma\right) \\
& =\operatorname{det}\left[\mu I_{N \ell}-\left(Q^{-1} \otimes I_{\ell}\right) \Gamma\left(Q \otimes I_{\ell}\right)\right] \\
& =\operatorname{det}\left[\mu I_{N \ell}-I_{N} \otimes A+\alpha J \otimes B\right] \\
& =\prod_{i=1}^{N} \operatorname{det}\left(\mu I_{\ell}-A+\alpha \lambda_{i} B\right) \\
& =\prod_{i=1}^{N}\left(\mu^{\ell}+\delta_{\ell-1}^{i} \mu^{\ell-1}+\cdots+\delta_{1}^{i} \mu+\alpha \lambda_{i} \gamma_{0}\right) .
\end{aligned}
$$

Proof of Lemma 5. It follows from (8) that there are $n_{0}$ zero eigenvalues of $\Phi$ that correspond to each zero eigenvalue of $L$. In fact, for every zero eigenvalue $\lambda_{i}$ of $L$; that is, $\lambda_{i}=0$,

$$
\begin{aligned}
\mu^{\ell}+ & \delta_{\ell-1}^{i} \mu^{\ell-1}+\cdots+\delta_{1}^{i} \mu+\lambda_{i} \gamma_{0} \\
= & \mu^{\ell}+\delta_{\ell-1}^{i} \mu^{\ell-1}+\cdots \\
& +\delta_{n_{0}+1}^{i} \mu^{n_{0}+1}+c_{n_{0}} \mu^{n_{0}} \\
= & \mu^{n_{0}}\left(\mu^{\ell-n_{0}}+\delta_{\ell-n_{0}-1} \mu^{\ell-n_{0}-1}\right. \\
& \left.+\cdots+\delta_{n_{0}+1} \mu+c_{n_{0}}\right),
\end{aligned}
$$

which implies that every zero eigenvalue of $L$ corresponds to $n_{0}$ zero eigenvalues of $\Phi$. It is directly to see that $L$ has at least one zero eigenvalue with an associated eigenvector $\mathbf{1}$, since $L \mathbf{1}=0$. Therefore, $\Phi$ has at least $n_{0}$ zero eigenvalues. Moreover, It follows from (8) again that $\Phi$ has exactly $n_{0}$ eigenvalues if and only if $L$ has exactly a simple zero eigenvalues.

Next, by a similar method from [16], let $s$ be an eigenvalue of $\Phi$ with associated eigenvector $q=\left[p_{1}^{T}, p_{2}^{T}, \ldots, p_{\ell}^{T}\right]^{T}$, where $p_{1}^{T}, p_{2}^{T}, \ldots, p_{\ell}^{T}$ are $N \times 1$ column vectors. Therefore,

$$
\begin{aligned}
\Phi q= & {\left[\begin{array}{ccccc}
0_{N} & I_{N} & 0_{N} & \cdots & 0_{N} \\
0_{N} & 0_{N} & I_{N} & \cdots & 0_{N} \\
\vdots & \vdots & \vdots & \vdots & \vdots \\
0_{N} & 0_{N} & 0_{N} & \cdots & I_{N} \\
-\alpha \gamma_{0} L & -\Delta_{1} & -\Delta_{2} & \cdots & -\Delta_{\ell-1}
\end{array}\right] } \\
& \times\left[\begin{array}{c}
p_{1} \\
p_{2} \\
\vdots \\
p_{\ell-1} \\
p_{\ell}
\end{array}\right]=s\left[\begin{array}{c}
p_{1} \\
p_{2} \\
\vdots \\
p_{\ell-1} \\
p_{\ell}
\end{array}\right],
\end{aligned}
$$

which implies that $p_{2}=s p_{1}, p_{3}=s p_{2}, \ldots, p_{\ell}=s p_{\ell-1}$, $-\alpha \gamma_{0} L p_{1}-c_{1} p_{2}-\alpha \gamma_{1} L p_{2}-\cdots-c_{\ell-1} p_{\ell}-\alpha \gamma_{\ell-1} L p_{\ell}=s p_{\ell}$; 
moreover, $-\alpha \sum_{k \in M_{2}} \gamma_{k} s^{k} L p_{1}-\sum_{j \in M_{1}} c_{j} s^{j} p_{1}=s^{\ell} p_{1}$. Let $\kappa=$ $\sum_{j \in M_{1}} c_{j} s^{j}+s^{\ell} /-\alpha \sum_{k \in M_{2}} \gamma_{k} s^{k}$; that is, $\kappa$ is an eigenvalue of $L$ with associated eigenvector $p_{1}$. If $L$ has a simple zero eigenvalue, without loss of generality, suppose that $\kappa=0$, then $\Phi$ has a eigenvalue $s=0$ (the algebraic multiplicity is $n_{0}$ ), so $p_{2}=p_{3}=\cdots=p_{\ell}=0$ and $q=\left[p_{1}^{T}, 0_{N}^{T}, \ldots 0_{N}^{T}\right]^{T}$, which is only one linearly independent eigenvector for $\Phi$ associated with eigenvalue zero; that is, the geometric multiplicity of zero eigenvalue of $\Phi$ is one.

It is easy to note that

$$
\begin{aligned}
& \Phi \frac{1}{\sqrt{N}} e_{1} \otimes \mathbf{1}= \frac{1}{\sqrt{N}}\left(A e_{1}\right) \otimes\left(I_{N} \mathbf{1}\right) \\
&-\frac{1}{\sqrt{N}}\left(B e_{1}\right) \otimes(L \mathbf{1})=0 \\
& \frac{1}{c_{n_{0}}}\left(\left[c_{1}, \ldots, c_{\ell-1}, 1\right] \otimes \mathbf{1}^{T}\right) \Phi \\
&=\frac{1}{c_{n_{0}}}\left(\left[c_{1}, \ldots, c_{\ell-1}, 1\right] A\right) \otimes\left(\mathbf{1}^{T} I_{N}\right) \\
&-\frac{1}{c_{n_{0}}}\left(\left[c_{1}, \ldots, c_{\ell-1}, 1\right] B\right) \otimes\left(\mathbf{1}^{T} L\right)=0 .
\end{aligned}
$$

Therefore, we obtain

$$
\begin{aligned}
& w_{r}=\frac{1}{\sqrt{N}}[1,0, \ldots, 0]_{\ell \times 1}^{T} \otimes \mathbf{1}, \\
& v_{l}=\frac{1}{c_{n_{0}}}\left[c_{1}, \ldots, c_{\ell-1}, 1\right] \otimes \mathbf{1}^{T} .
\end{aligned}
$$

\section{Conflict of Interests}

The authors declare that there is no conflict of interests regarding the publication of this paper.

\section{Acknowledgments}

This work was jointly supported by the National Natural Science Foundation of China under Grant no. 61304169, the Natural Science Foundation of the Jiangsu Higher Education Institutions under Grant no. 13KJB110022, the Natural Science Foundation of Jiangsu Province of China under Grant no. BK20130857 and the Scientific Research Foundation of Nanjing University of Posts and Telecommunications under Grant no. NY213052.

\section{References}

[1] T. Vicsek, A. Czirk, E. Ben-Jacob, I. Cohen, and O. Shochet, "Novel type of phase transition in a system of self-driven particles," Physical Review Letters, vol. 75, no. 6, pp. 1226-1229, 1995.

[2] A. Jadbabaie, J. Lin, and A. S. Morse, "Coordination of groups of mobile autonomous agents using nearest neighbor rules," IEEE Transactions on Automatic Control, vol. 48, no. 6, pp. 988-1001, 2003.
[3] R. Olfati-Saber and R. M. Murray, "Consensus problems in networks of agents with switching topology and time-delays," IEEE Transactions on Automatic Control, vol. 49, no. 9, pp. 15201533, 2004.

[4] W. Ren and R. W. Beard, "Consensus seeking in multi-agent systems under dynamically changing interaction topologies," IEEE Transactions on Automatic Control, vol. 50, no. 5, pp. 655661, 2005.

[5] Z. Meng, W. Ren, Y. Cao, and Z. You, "Leaderless and leaderfollowing consensus with communication and input delays under a directed network topology," IEEE Transactions on Systems, Man, and Cybernetics B, vol. 41, no. 1, pp. 75-88, 2011.

[6] G. Xie and L. Wang, "Consensus control for a class of networks of dynamic agents," International Journal of Robust and Nonlinear Control, vol. 17, no. 10-11, pp. 941-959, 2007.

[7] W. Ren, "On consensus algorithms for double-integrator dynamics," IEEE Transactions on Automatic Control, vol. 58, no. 6, pp. 1503-1509, 2008.

[8] G. Wen, Z. Duan, W. Yu, and G. Chen, "Consensus of secondorder multi-agent systems with delayed nonlinear dynamics and intermittent communications," International Journal of Control, vol. 86, no. 2, pp. 322-331, 2013.

[9] W. Yu, G. Chen, M. Cao, and J. Kurths, "Second-order consensus for multi-agent systems with directed topologies and nonlinear dynamics," IEEE Transactions on Systems, Man, and Cybernetics B, vol. 40, no. 3, pp. 881-891, 2010.

[10] W. Yu, G. Chen, and M. Cao, "Some necessary and sufficient conditions for second-order consensus in multi-agent dynamical systems," Automatica, vol. 46, no. 6, pp. 1089-1095, 2010.

[11] Q. Song, J. Cao, and W. Yu, "Second-order leader-following consensus of nonlinear multi-agent systems via pinning control," Systems \& Control Letters, vol. 59, no. 9, pp. 553-562, 2010.

[12] W. Zhu and D. Cheng, "Leader-following consensus of secondorder agents with multiple time-varying delays," Automatica, vol. 46, no. 12, pp. 1994-1999, 2010.

[13] G. Wen, Z. Duan, W. Yu, and G. Chen, "Consensus in multiagent systems with communication constraints," International Journal of Robust and Nonlinear Control, vol. 22, no. 2, pp. 170$182,2012$.

[14] G. Wen, Z. Duan, W. Yu, and G. Chen, "Consensus of multiagent systems with nonlinear dynamics and sampled-data information: a delayed-input approach," International Journal of Robust and Nonlinear Control, vol. 23, no. 6, pp. 602-619, 2013.

[15] Z. Li, Z. Duan, G. Chen, and L. Huang, "Consensus of multiagent systems and synchronization of complex networks: a unified viewpoint," IEEE Transactions on Circuits and Systems I, vol. 57, no. 1, pp. 213-224, 2010.

[16] W. Ren, K. L. Moore, and Y. Chen, "High-order and model reference consensus algorithms in cooperative control of multivehicle systems," Journal of Dynamic Systems, Measurement and Control, vol. 129, no. 5, pp. 678-688, 2007.

[17] F. Jiang, L. Wang, and Y. Jia, "Consensus in leaderless networks of high-order-integrator agents," in Proceedings of the American Control Conference (ACC '09), pp. 4458-4462, St. Louis, Mo, USA, June 2009.

[18] W. He and J. Cao, "Consensus control for high-order multiagent systems," IET Control Theory \& Applications, vol. 5, no. 1, pp. 231-238, 2011.

[19] F. Xiao and L. Wang, "Consensus problems for high-dimensional multi-agent systems," IET Control Theory \& Applications, vol. 1, no. 3, pp. 830-837, 2007. 
[20] N. Cai, J.-X. Xi, and Y.-S. Zhong, "Swarm stability of high-order linear time-invariant swarm systems," IET Control Theory \& Applications, vol. 5, no. 2, pp. 402-408, 2011.

[21] P. Lin and Y. Jia, "Consensus of a class of second-order multiagent systems with time-delay and jointly-connected topologies," IEEE Transactions on Automatic Control, vol. 55, no. 3, pp. 778-784, 2010.

[22] C. Liu and F. Liu, "Consensus problem of coupled dynamic agents with communication delay," in Proceedings of the 29th Chinese Control Conference (CCC '10), pp. 4501-4505, Beijing, China, July 2010.

[23] X. Liu, W. Lu, and T. Chen, "Consensus of multi-agent systems with unbounded time-varying delays," IEEE Transactions on Automatic Control, vol. 55, no. 10, pp. 2396-2401, 2010.

[24] Y. G. Sun, L. Wang, and G. Xie, "Average consensus in networks of dynamic agents with switching topologies and multiple timevarying delays," Systems \& Control Letters, vol. 57, no. 2, pp. 175$183,2008$.

[25] W. Yu, G. Chen, and W. Ren, "Delay-induced quasi-consensus in multi-agent dynamical systems," in Proceedings of the 29th Chinese Control Conference (CCC '10), pp. 4566-4571, Beijing, China, July 2010.

[26] C. Godsil and G. Royle, Algebraic Graph Theory, Springer, New York, NY, USA, 2001. 


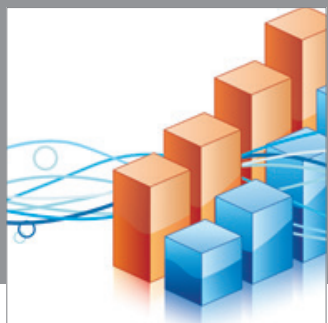

Advances in

Operations Research

mansans

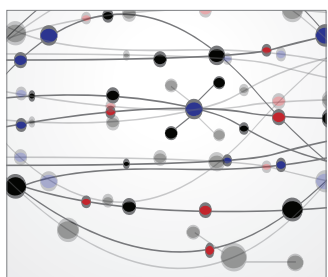

The Scientific World Journal
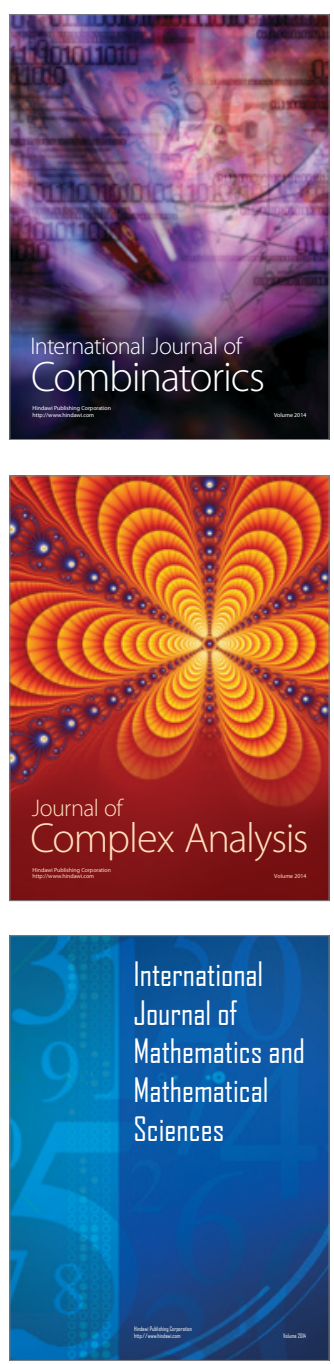
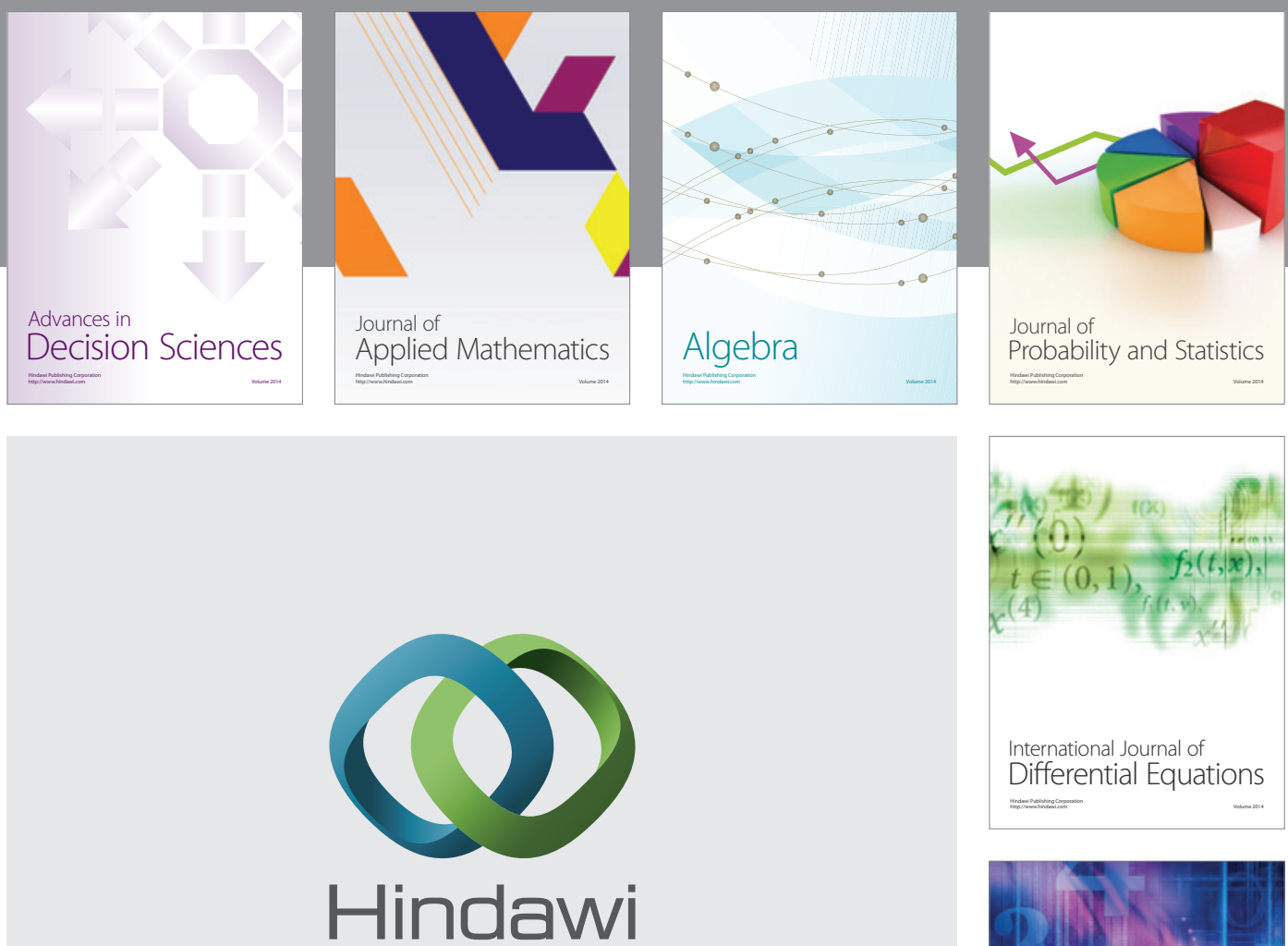

Submit your manuscripts at http://www.hindawi.com
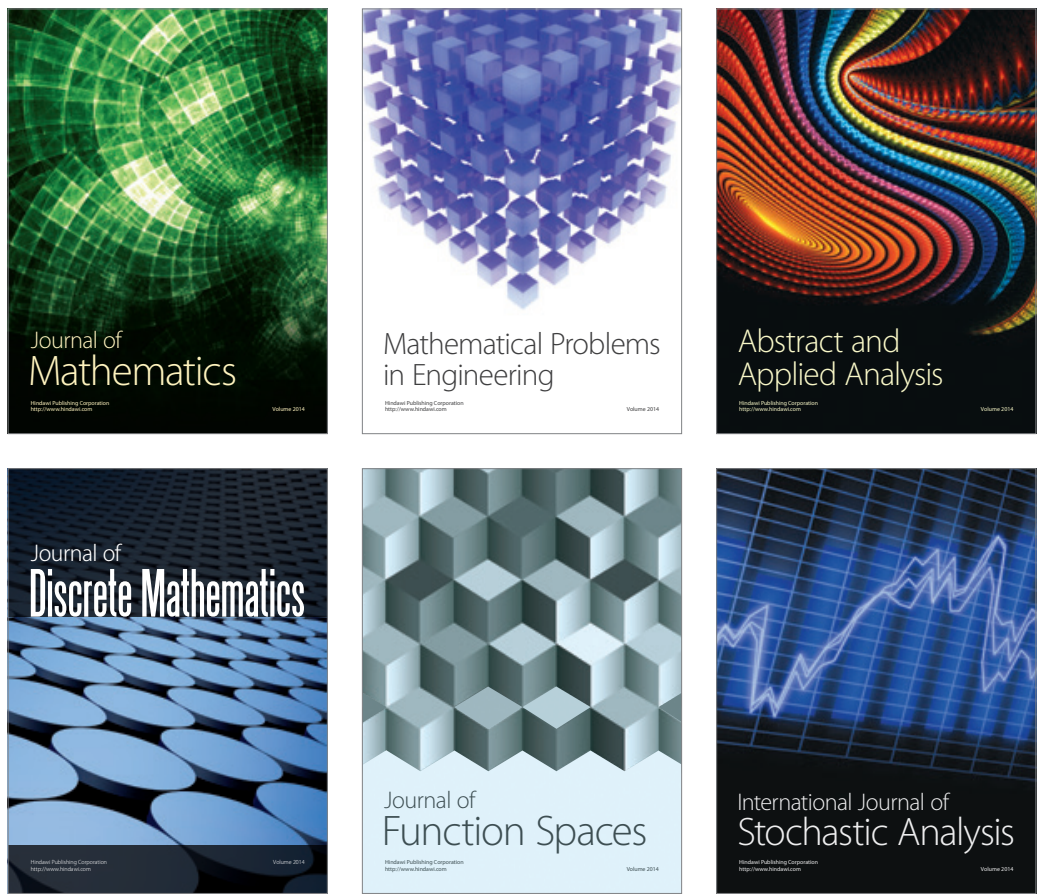

Journal of

Function Spaces

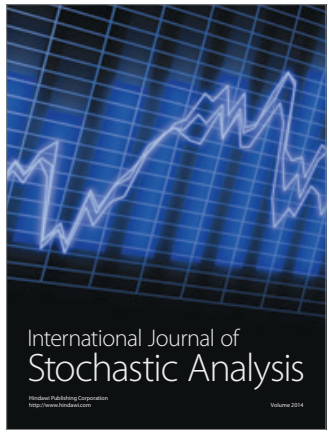

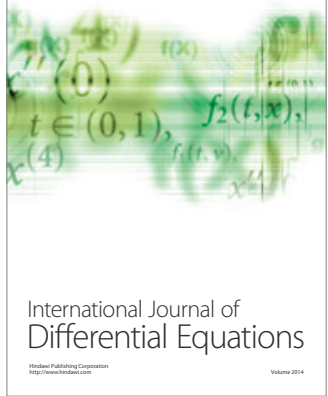
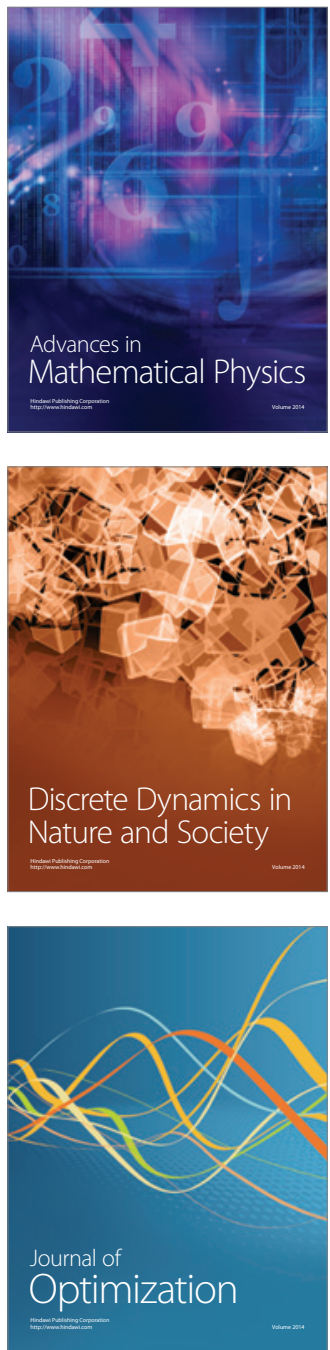